\title{
Chronic Cholestatic Liver Injury Attributable to Vedolizumab
}

\author{
Jonathan G. Stine ${ }^{* 1}$, Jennifer Wang ${ }^{2}$ and Brian W. Behm ${ }^{1}$ \\ ${ }^{1}$ Division of Gastroenterology \& Hepatology, Department of Medicine, University of Virginia, Charlottesville, VA, USA; \\ ${ }^{2}$ Department of Medicine, University of Virginia, Charlottesville, VA, USA
}

\begin{abstract}
Drug-induced liver injury is a rare but clinically important diagnosis. Vedolizumab is an $\alpha 4 \beta 7$ integrin inhibitor recently approved for use in patients with moderate-to-severe inflammatory bowel disease. Cases of hepatoxicity due to vedolizumab in the pre-marketing stage were rare, and all cases resolved upon drug withdrawal. We present here the first reported case of hepatotoxicity attributable to vedolizumab, which despite drug cessation persisted with chronic cholestatic liver injury.

(C) 2016 The Second Affiliated Hospital of Chongqing Medical University. Published by XIA \& HE Publishing Inc. All rights reserved.
\end{abstract}

\section{Introduction}

Drug-induced liver injury (DILI) has been associated with over 1,100 drugs and herbal medications ${ }^{1}$ and can present with all forms of acute and chronic liver diseases. ${ }^{2,3}$ Most cases of DILI are self-limiting and resolve with prompt identification and cessation of the offending agent; ${ }^{2,3}$ however, chronic DILI, defined as persistent abnormalities in liverassociated enzymes at 3-6 months after drug withdrawal, ${ }^{3,4}$ is becoming increasingly recognized. ${ }^{5}$

Vedolizumab (VDZ) is an $\alpha 4 \beta 7$ integrin inhibitor recently approved for use in patients with moderate-to-severe Crohn's disease and ulcerative colitis (UC). ${ }^{6}$ In general, VDZ is well tolerated; serious adverse events (SAEs) have been reported in $7-12 \%$ of patients in the pre-marketing phase III trials, ${ }^{7}$ but this range is similar to that of the SAEs experienced by the placebo groups (4-9\%). Liver injury has rarely been reported with VDZ. Review of the aggregate pre-marketing data showed that among the 3,326 patients exposed to VDZ in phase III, controlled or open-label studies, $<2 \%$ experienced elevations in aminotransferases $>3 \times$ the upper limit of normal. ${ }^{6,7}$ Four cases of acute DILI, with or without cholestasis, have been reported and these resolved with drug

Keywords: Hepatotoxicity; Integrin inhibitor; Primary sclerosing cholangitis; Inflammatory bowel disease.

Abbreviations: DILI, drug-induced liver injury; VDZ, Vedolizumab; UC, ulcerative colitis; SAEs, serious adverse events; PSC, primary sclerosing cholangitis; IBD, inflammatory bowel disease; AST, aminotransferase; ALT, alanine aminotransferase; TB, total bilirubin; INR, international normalized ratio; ULN, upper limit of normal.

Received: 19 April 2016; Revised: 03 June 2016; Accepted: 05 June 2016

DOI: $10.14218 / \mathrm{JCTH} .2016 .00018$.

*Correspondence to: Jonathan G. Stine, Division of Gastroenterology \& Hepatology, University of Virginia, JPA and Lee Street, MSB 2145, PO Box 800708, Charlottesville, VA 22908-0708, USA. Tel: +1-434-243-7741, Fax: +1-434-2447529, E-mail: Jgs9f@virginia.edu discontinuation and/or administration of corticosteroids. ${ }^{6}$ Consequently, the drug-package insert states that VDZ should be discontinued in patients with jaundice or other evidence of significant liver injury. ${ }^{6}$ A recent post-marketing multicenter experience in 172 patients failed to find a single hepatotoxic event, and the authors concluded that VDZ is safe and well tolerated in clinical practice. ${ }^{8}$

We present herein the first case of post-marketing hepatotoxicity attributable to VDZ that presented with acute cholestasis and progressed to chronic liver injury despite prompt drug withdrawal and corticosteroid administration.

\section{Case reports}

A 23-year-old Caucasian male with pancolonic UC diagnosed at age 19 and concurrent primary sclerosing cholangitis (PSC) diagnosed at age 22 was evaluated at our institution in the Digestive Health outpatient clinic. His prior inflammatory bowel disease (IBD) therapy included 5-aminosalicylate medications, corticosteroids, azathioprine and biologic agents (infliximab and adalimumab) prescribed by his gastroenterologist following the initial diagnosis, but achieved no clinical or endoscopic remission. Examination at the time of his initial adult consultation did not reveal any stigmata of advanced liver disease. Pre-treatment liver biochemistries were notable for aspartate aminotransferase (AST) at $31 \mathrm{U} / \mathrm{L}$, alanine aminotransferase (ALT) at $33 \mathrm{U} / \mathrm{L}$, alkaline phosphatase at $648 \mathrm{U} / \mathrm{L}(61 \%$ heat stabile; normal range $25-40 \%)$, total bilirubin (TB) at $1.4 \mathrm{mg} / \mathrm{dL}$ and albumin at $2.9 \mathrm{~g} / \mathrm{dL}$. Gamma glutamyltransferase was $130 \mathrm{U} / \mathrm{L}$ (normal < $55 \mathrm{U} / \mathrm{L}$ ). Other notable lab results included platelet count of $525 \mathrm{k} / \mu \mathrm{L}$, international normalized ratio (INR) of 1.1 , sedimentation rate of $24 \mathrm{~mm} / \mathrm{hr}$ (normal $0-15 \mathrm{~mm} / \mathrm{hr}$ ) and C-reactive protein at $0.1 \mathrm{~g} / \mathrm{dL}$ (normal $<0.5 \mathrm{~g} / \mathrm{dL}$ ).

After the patient's initial consultation, he was initiated on VDZ and received three doses of $300 \mathrm{mg}$ intravenous infusions at weeks 0,2 and 6 . After the third infusion, the patient developed insidious onset of jaundice and fatigue. Importantly, he had never experienced overt jaundice due to PSC previously. Laboratory tests at this time were remarkable for AST at $52 \mathrm{U} / \mathrm{L}$, ALT at $27 \mathrm{U} / \mathrm{L}$, alkaline phosphatase at $370 \mathrm{U} / \mathrm{L}$, TB $13.3 \mathrm{mg} / \mathrm{dL}$, and conjugated bilirubin $9.3 \mathrm{mg} / \mathrm{dL}$. The $\mathrm{R}$ ratio was calculated [(ALT/upper limit of normal (ULN))/(alkaline phosphatase/ULN)] and found to be 1.1, indicating cholestatic liver injury ( $R$ ratio $<2$ is diagnostic).

Magnetic resonance imaging of the abdomen, including magnetic resonance cholangiopancreatography scanning, did not reveal any dominant stricture and the findings were unchanged compared to the index images that were taken one year prior, at the time of the PSC diagnosis. A comprehensive hepatitis viral serology panel was unrevealing. 
Stine J.G. et al: Vedolizumab hepatotoxicity

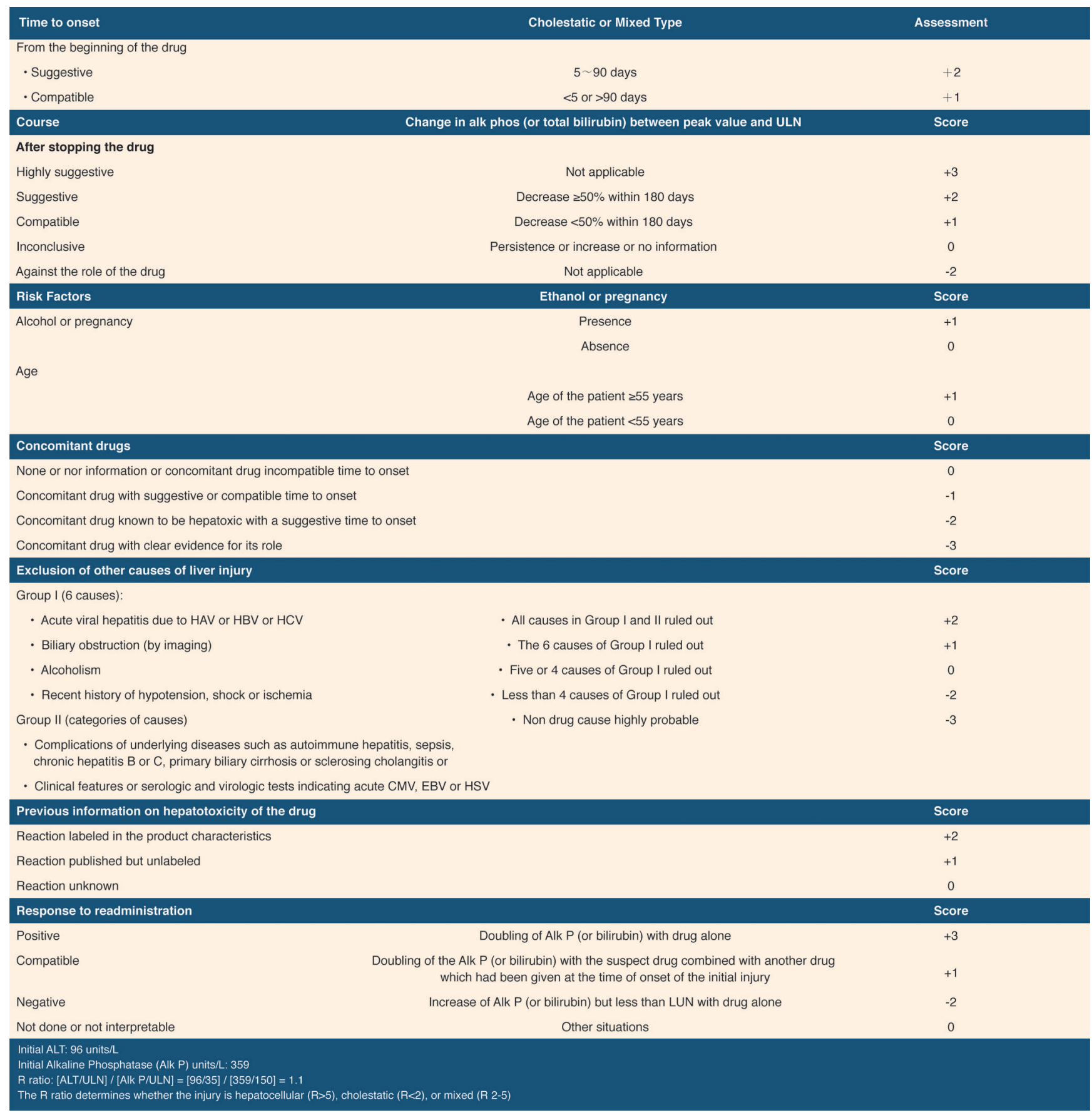

Fig. 1. Roussel Uclaf Causality Assessment Method (RUCAM) scoring. ${ }^{13}$

The patient denied any alcohol or toxin exposure. Testing for autoimmune hepatitis and hemochromatosis screening was negative. The Roussel Uclaf Causality Assessment Method (commonly known as RUCAM), which uses seven different criteria to quantify the strength of association between a liver injury and the medication, implicated VDZ as the offending agent with a score of 7 (probable liver injury) (Fig. 1).

VDZ was subsequently discontinued after the third infusion. Six weeks after the patient's last dose of VDZ, he experienced an increase in bowel frequency with associated bleeding and was started on prednisone. Lab results from several weeks after the corticosteroid administration showed marked improvement accompanying the continued corticosteroid use, with TB at $4.9 \mathrm{~g} / \mathrm{dL}$ and alkaline phosphatase at $485 \mathrm{U} / \mathrm{L}$. The patient's jaundice and fatigue also improved. Corticosteroid therapy was continued for the IBD, and antiTNF therapy with adalimumab was resumed 4 months after the last dose of VDZ; the patient experienced intervals of improvement in his bowel symptoms but not complete clinical remission. 
Stine J.G. et al: Vedolizumab hepatotoxicity

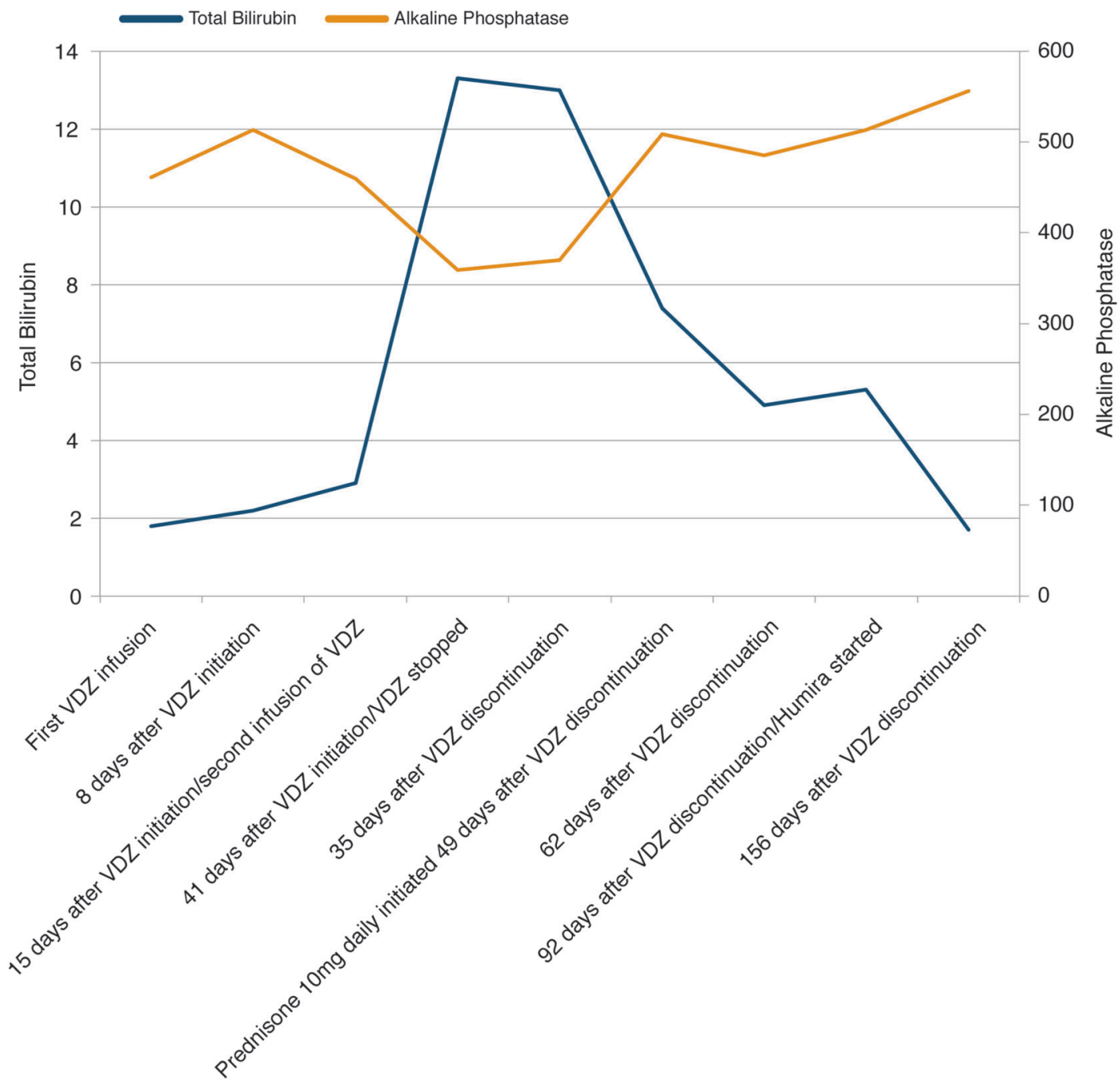

Fig. 2. Natural history of liver-associated enzymes in the course of vedolizumab treatment.

Importantly, the patient's cholestatic liver injury persisted 5 months following the drug withdrawal, meeting criteria for chronic DILI with TB at $1.7 \mathrm{~g} / \mathrm{dL}$ and alkaline phosphatase at $556 \mathrm{U} / \mathrm{L}$. Pre-DILI diagnosis lab results were compared to post-DILI lab results within a 6 -week window in each period by using a paired $t$-test. TB levels were significantly different (pre-DILI: $2.3 \pm 0.6 \mathrm{~g} / \mathrm{dL}$ vs. post-DILI: $10.4 \pm 4.2 \mathrm{~g} / \mathrm{dL}$, $p=0.037$ ) while AST and ALT were not significantly different by the statistical testing (Table 1 ). Figure 2 shows the graphic trend in the patient's liver-associated enzymes. Informed consent was obtained from the patient.

\section{Discussion}

While pre-marketing data suggests that hepatotoxicity in the setting of VDZ use for IBD is a rare and self-limiting event, with or without cholestasis, ${ }^{6,7}$ we present the first postmarketing case of chronic DILI attributable to VDZ use. Chronic liver injury from medications is becoming increasingly recognized, ${ }^{6}$ with prevalence rates approaching $20 \%$ based upon data from the Drug-Induced Liver Injury Network (DILIN). ${ }^{3}$ Patients with cholestatic injury, such as our patient, appear to be at increased risk for progressing to chronic injury. ${ }^{3,10}$ Adherence to appropriate prescription regimens remains one of the most important aspects for preventing DILI in patients with underlying chronic liver disease. ${ }^{9}$ The drug package insert for VDZ states that elevations of liver-associated enzymes have been reported in patients receiving VDZ and that in the absence of biliary obstruction VDZ should be discontinued in patients with jaundice or other evidence of significant liver injury. ${ }^{6}$

Whether or not VDZ may worsen bile duct injury in the setting of PSC through an unknown mechanism remains to be determined. In general, PSC is not thought to place patients at increased risk for DILI. ${ }^{2}$ Rather, secondary sclerosing cholangitis typically occurs as a sequela of chronic druginduced bile duct injury. ${ }^{10} \mathrm{~A}$ solitary case report of DILI in the setting of certirizine use in a patient with PSC has been reported. ${ }^{11}$ Based on the available evidence, in combination with our isolated case report, we cannot recommend avoiding 


\begin{tabular}{|c|c|c|c|c|}
\hline & AST & ALT & Total bilirubin & ALP \\
\hline First VDZ infusion & 31 & 33 & 1.8 & 461 \\
\hline 8 days after VDZ initiation & 63 & 62 & 2.2 & 513 \\
\hline 15 days after VDZ initiation/second infusion of VDZ & * & * & 2.9 & 460 \\
\hline 41 days after VDZ initiation/VDZ discontinued & 52 & 27 & 13.3 & 359 \\
\hline 35 days after VDZ discontinuation & 48 & 25 & 13 & 370 \\
\hline 49 days after VDZ discontinuation/prednisone ( $10 \mathrm{mg}$ daily) initiated & 55 & 16 & 7.4 & 509 \\
\hline 62 days after VDZ discontinuation & 78 & 52 & 4.9 & 485 \\
\hline 92 days after VDZ discontinuation/Humira started & 50 & 33 & 5.3 & 513 \\
\hline 156 days after VDZ discontinuation & 55 & 77 & 1.7 & 556 \\
\hline
\end{tabular}

*The data of aspartate aminotransferase (AST) and alanine aminotransferase (ALT) at 15 days after VDZ initiation were not collected, and therefore not reported. ALP, alkaline phosphatase.

VDZ in IBD patients with concomitant PSC; however, monitoring hepatic enzymes during VDZ therapy is warranted.

In general, treatment options for DILI are limited. Treatment is centered around prompt identification and removal of the offending drug. ${ }^{12}$ While corticosteroids were administered to several patients in the pre-marketing trials who experienced hepatotoxic events, the efficacy of the use of corticosteroids in treating DILI remains largely anecdotal. ${ }^{13}$ Immunosuppressive therapy may be considered on a case-bycase basis, where autoimmune-like DILI features, including the presence of auto-antibodies or liver histology supporting this diagnosis, are present and cessation of the drug does not lead to complete resolution of injury. ${ }^{5}$

In this case, corticosteroids were administered to address worsening IBD activity and not as treatment for DILI, but they may have hastened clinical improvement following the hepatotoxic event. On the other hand, corticosteroids and adalimumab have also been implicated in cholestatic liver injury. In the present case, the patient had previously been on these agents for his UC, without showing evidence of liver injury. In addition, the patient's total bilirubin continued to improve after initiation of corticosteroids and adalimumab, which would argue against these therapies leading to persistent chronic liver injury.

While this case has several limitations, including an incomplete evaluation for other chronic liver diseases (namely, alpha-1 antitrypsin deficiency or Wilson's disease) and a lack of liver biopsy as a confirmatory study, a high-index of suspicion for the development of hepatotoxicity in patients with IBD being treated with VDZ seems necessary based on our report, nonetheless.

\section{Acknowledgement}

The research reported in this publication was supported by the National Institute of Diabetes and Digestive and Kidney Diseases of the National Institutes of Health under award number T32DK007769. The content is solely the responsibility of the authors and does not necessarily represent the official views of the National Institutes of Health.

\section{Conflict of interest}

None

\section{Author contributions}

Manuscript design, manuscript writing, final approval (JGS), manuscript writing, final approval (JW), manuscript design, final approval (BWB).

\section{References}

[1] Reuben A, Koch DG, Lee WM. Drug-induced acute liver failure: results of a U.S. multicenter, prospective study. Hepatology 2010;52:2065-2076. doi: 10.1002/hep.23937.

[2] Chalasani N, Fontana RJ, Bonkovsky HL, Watkins PB, Davern T, Serrano J, et al. Causes, clinical features, and outcomes from a prospective study of drug-induced liver injury in the United States. Gastroenterology 2008;135: 1924-1934, 1934.e1921-1924.

[3] Chalasani N, Bonkovsky HL, Fontana R, Lee W, Stolz A, Talwalkar J, et al. Features and outcomes of 889 patients with drug-induced liver injury: the DILIN prospective study. Gastroenterology 2015;148:1340-1352.e1347. doi: $10.1053 /$ j.gastro.2015.03.006.

[4] Andrade RJ, Lucena MI, Kaplowitz N, Garcia-Munoz B, Borraz Y, Pachkoria K, et al. Outcome of acute idiosyncratic drug-induced liver injury: Long-term follow-up in a hepatotoxicity registry. Hepatology 2006;44:1581-1588. doi: 10.1002/hep.21424.

[5] Stine JG, Chalasani N. Chronic liver injury induced by drugs: a systematic review. Liver Int 2015;35:2343-2353. doi: 10.1111/liv.12958.

[6] Vedolizumab Drug-package insert. Accessed 15 Oct 2015. Available at http://www.accessdata.fda.gov/drugsatfda_docs/label/2014/125476s000 lbl.pdf.

[7] Colombel JE, Loftus EV, Siegel CA, Lewis J, Smyth MD, Sankoh S, et al. Efficacy of vedolizumab with concomitant corticosteroid or immunomodulator use in patients with ulcerative colitis from GEMINI 1. Gastroenterology 2015;148:S277-S278. doi: 10.1016/s0016-5085(15)30911-2.

[8] Shelton E, Allegretti JR, Stevens B, Lucci M, Khalili H, Nguyen DD, et al. Efficacy of vedolizumab as induction therapy in refractory IBD patients: a multicenter cohort. Inflamm Bowel Dis 2015;21:2879-2885. doi: 10. 1097/MIB.0000000000000561.

[9] Lewis JH, Stine JG. Review article: prescribing medications in patients with cirrhosis - a practical guide. Aliment Pharmacol Ther 2013;37:1132-1156. doi: 10.1111 /apt.12324.

[10] Gudnason HO, Björnsson HK, Gardarsdottir M, Thorisson HM, Olafsson S, Bergmann OM, et al. Secondary sclerosing cholangitis in patients with drug-induced liver injury. Dig Liver Dis 2015;47:502-507. doi: 10.1016/j. dld.2015.03.002.

[11] Jurawan R, Smith A. Severe hepatitis in a primary sclerosing cholangitis patient receiving recent cetirizine therapy. N Z Med J 2010;123:106-107.

[12] Stine JG, Lewis JH. Current and future directions in the treatment and prevention of drug-induced liver injury: a systematic review. Expert Rev Gastroenterol Hepatol 2016;10:517-536. doi: 10.1586/17474124.2016.1127756.

[13] Stine JG, Intagliata N, Shah NL, Argo CK, Caldwell SH, Lewis JH, et al. Hepatic decompensation likely attributable to simeprevir in patients with advanced cirrhosis. Dig Dis Sci 2015;60:1031-1035. doi: 10.1007/ s10620-014-3422-x. 